\title{
Adrenocortical Oncocytoma Presenting with Gynaecomastia
}

\author{
Than Than Aye, ${ }^{1}$ Phone Myint, ${ }^{2}$ Kyar Nyo Soe Myint ${ }^{1}$ \\ ${ }^{1}$ Department of Endocrinology, University of Medicine 2, Yangon, Myanmar \\ ${ }^{2}$ Department of Urology, University of Medicine 2, Yangon, Myanmar
}

\begin{abstract}
Adrenal oncocytomas are rare adrenal neoplasms which are usually large and nonfunctioning. Most of them are benign. We report a rare case of adrenal oncocytoma presenting with bilateral gynaecomastia and hypogonadism in $67-$ year-old male from Myanmar who had elevated estradiol levels. He successfully underwent an adrenalectomy and the histologic diagnosis was an oncocytoma. The immunohistochemical (IHC) staining was positive for neuron specific enolase (NSE), vimentin and synaptophysin. Feminizing adrenal oncocytoma has not been reported in literature.
\end{abstract}

Key words: gynaecomastia, feminizing, adrenal tumour, oncocytoma

\section{INTRODUCTION}

Oncocytoma is a rare tumour of the adrenal gland. With only 51 cases reported in the literature to date, adrenocortical oncocytoma is an exceedingly rare pathological variant of adrenal neoplasm. ${ }^{1}$ The typical distinguishing histological feature of oncocytoma is the presence of epithelial cells with abundant mitochondria within a granular eosinophilic cytoplasm. ${ }^{2}$ Most adrenocortical oncocytic neoplasms are benign, nonfunctioning and are detected incidentally. We report a case of a patient with a functioning adrenal oncocytic neoplasm who presented with bilateral gynaecomastia.

\section{CASE}

A 76-year-old male from Myanmar with progressive enlargement of his left breast, and later the right breast over a 4 month duration consulted at the Endocrine Unit of the North Okkala Pa General Hospital, Myanmar. One month prior to consult, he also had pain over his right breast. There was also occassional dull pain on the right side of the abdomen. He presented with features of hypogonadism such as reduced libido, reduced body hair and erectile dysfunction. There was no testicular enlargement, no history of malignancies, hepatic, renal, endocrine diseases and no history of alcohol dependency.

The patient had hypertension for 10 years, well controlled with amlodipine $5 \mathrm{mg}$ once a day. Physical examination was unremarkable, apart from bilateral gynaecomastia with areolar tissue $2 \times 3 \mathrm{~cm}$ in both breasts. Chest and abdominal examination were unremarkable. Blood for complete

e-ISSN 2308-118X

Printed in the Philippines

Copyright $(0) 2015$ by the JAFES

Received March 30, 2015. Accepted May 15, 2015.

http://dx.doi.org/10.15605/jafes.030.01.08 picture, renal function, serum electrolytes were all within normal limits. Ultrasound of the abdomen revealed a well defined, hyperechoic mass in the upper pole of the right kidney (adrenal tumour) measuring $6.2 \times 5.1 \times 5 \mathrm{~cm}$. The liver and hepatobiliary tree appeared normal. Ultrasound of the testes showed bilateral atrophic testes. The right adrenal mass was subsequently confirmed by contrast enhanced computerized tomography (CECT) of the abdomen, which showed an oval shaped lesion measuring $7.8 \times 6.6 \times 7.4 \mathrm{~cm}$ (Figure 1a-b). The left adrenal gland also had a $1.3 \mathrm{~cm}$ nodule. Because of his gynaecomastia and hypogonadism, testosterone, estradiol, human chorionic gonadotrophin ( $\beta$-hCG), lutenizing hormone and prolactin were done to evaluate the cause. (Table 1). Adrenocortical hormones were measured by doing an 8 am cortisol, urinary metanephrine and normetanephrine all of which were normal. He subsequently underwent right adrenalectomy by laparotomy.

\begin{tabular}{lll}
\multicolumn{2}{l}{ Table 1. Laboratory evaluation } \\
\hline Preoperative & Patient & Normal range \\
\hline Testosterone & $4.9 \mathrm{nmol} / \mathrm{L}$ & $8.4-28.7 \mathrm{nmol} / \mathrm{L}$ \\
$\beta$-hCG & $<0.1 \mathrm{U} / \mathrm{L}$ & $0-5 \mathrm{U} / \mathrm{L}$ \\
Estradiol & $1160.26 \mathrm{pmol} / \mathrm{L}$ & $0-191 \mathrm{pmol} / \mathrm{L}$ \\
Prolactin & $574 \mathrm{mU} / \mathrm{L}$ & $60-620 \mathrm{mU} / \mathrm{L}$ \\
$\mathrm{LH}$ & $3.1 \mathrm{U} / \mathrm{L}$ & $3-8 \mathrm{U} / \mathrm{L}$ \\
Cortisol $(8 \mathrm{am})$ & $278.08 \mathrm{nmol} / \mathrm{L}$ & $180-620 \mathrm{nmol} / \mathrm{L}$ \\
Urinary metanephrine & $1.33 \mu \mathrm{mol} / 24 \mathrm{hr}$ & $1.9 \mu \mathrm{mol} / 24 \mathrm{hr}$ \\
Urinary normetanephrine & $2.4 \mu \mathrm{mol} / 24 \mathrm{hr}$ & $4.5 \mu \mathrm{mol} / 24 \mathrm{hr}$ \\
\hline Postoperative & \\
\hline Testosterone & $3.8 \mathrm{nmol} / \mathrm{L}$ \\
Estradiol & $62.41 \mathrm{pmol} / \mathrm{L}$ & $8.4-28.7 \mathrm{nmol} / \mathrm{L}$ \\
\hline
\end{tabular}

The operative specimen was a large, reddish-brown mass measuring $8.5 \times 6.5 \times 4.5 \mathrm{~cm}$ (216 grams) well, encapsulated tumor with a yellow-brown, lobulated, soft

Corresponding author: Prof. Than Than Aye, MBBS

President, Myanmar Society of Endocrinology and Metabolism

University of Medicine 2, Yangon, Myanmar

No.18, Kan Street, Hlaing Township,

Yangon, Myanmar

Tel. No.: 959-5060558

E-mail: profthanthanaye@gmail.com 
ONLINE FIRST | May 21, 2015 | http://dx.doi.org/10.15605/jafes.030.01.08

surface. An area of haemorrhage and cystic areas filled with old blood is present. Microscopic sections showed a well-encapsulated tumour composed of large polyhedral cells arranged in sheets and lobules. The cells have central to eccentric round vesicular nuclei and abundant brightly eosinophilic granular cytoplasm (Figure 2a-b). Nuclear pleomorphism and occasional multinucleated giant cells are noted. There are rare mitotic figures seen. Invasion of the fibrous capsule, lymphovascular invasion and infiltration of the surrounding tissue are not seen. Immunohistochemical stains were strongly positive for vimentin and neuron specific enolase (NSE), and weakly positive for synaptophysin (Figure 3a-d). One week after the operation, his estradiol levels decreased, no features of distance metastasis were noted and he will be followed up every 6 months to check for recurrence.

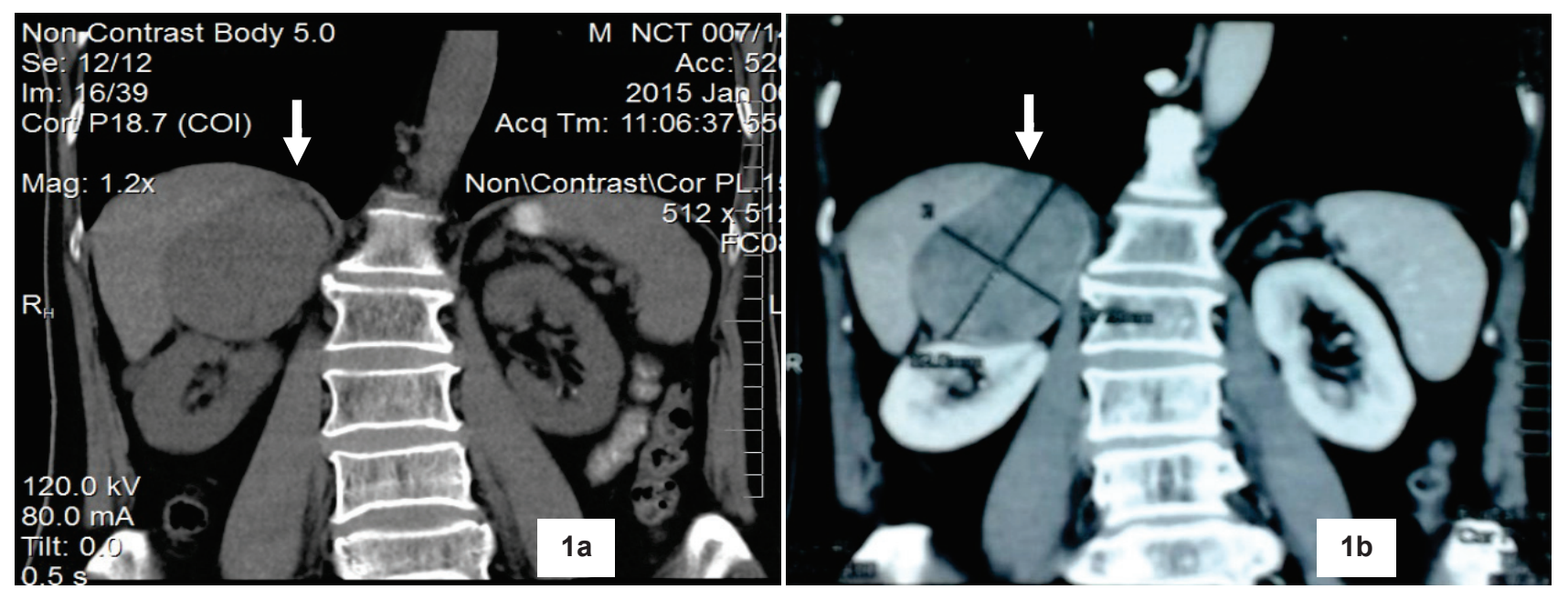

Figures 1a-b. Abdominal scan showing right adrenal mass. 1a. Without contrast. 1b. With contrast enhancement.
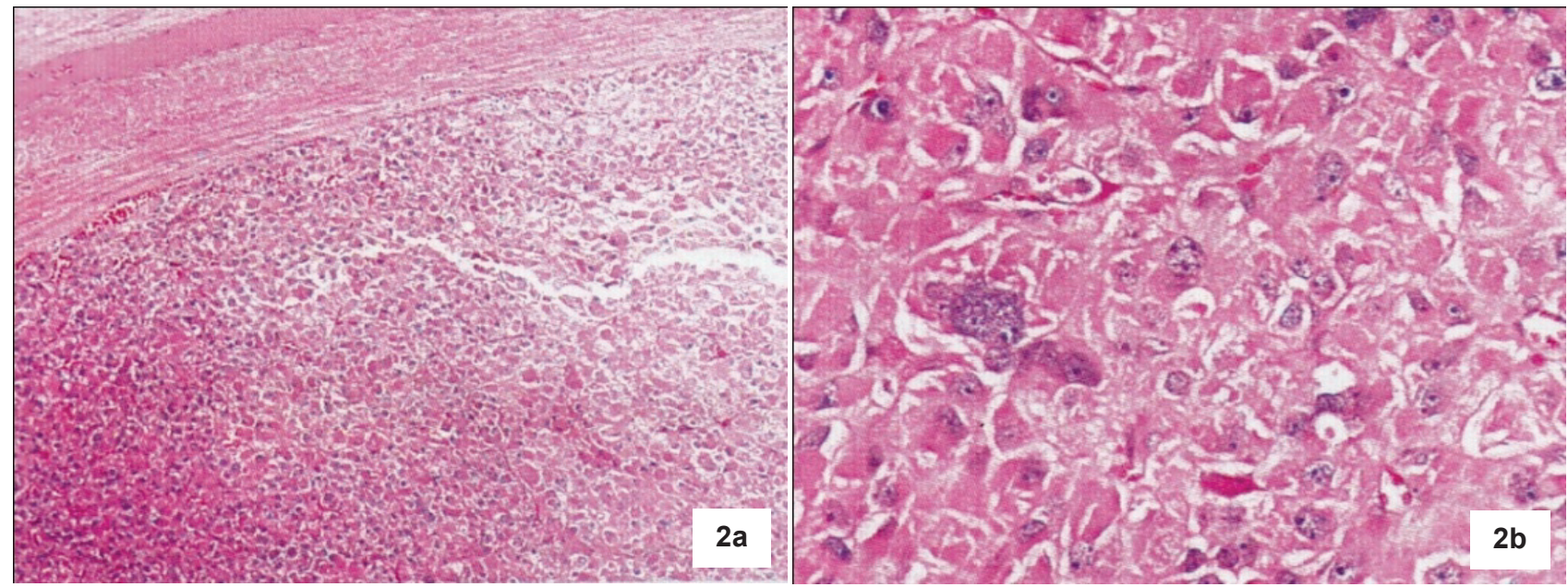

Figures 2a-b. 2a. Adrenocortical oncocytoma, well encapsulated tumour with granular basophilic cytoplasm (H\&E x 10). 2b. Tumor cells contain densely granular eosinophilic cytoplasm. Nuclear pleomorphism is noted in a few cells (H\&E x 400).

\section{DISCUSSION}

Oncocytic neoplasms are mostly found in the kidney, thyroid and salivary gland but are rare in the adrenal glands. There is female predominance with mean age of 46 years and more common on the left adrenal, although our case is a 76-year-old man with a right adrenal oncocytoma. Most of these tumours are non-functioning and are incidentally detected on routine checkup. ${ }^{3}$ Functioning tumours are a rare entity. Adrenal oncocytoma presenting with virilization. ${ }^{4}$ Cushing's syndrome ${ }^{5}$ and mimicking a pheochromocytoma ${ }^{6}$ were reported but we have not found any published cases with feminizing syndrome. Adrenal carcinoma was considered in the differential diagnosis.

There is no single histological parameter that is predictive of clinical outcome and biologic behavior of adrenal oncocytomas. The most widely used histologic criteria for the diagnostic categorization of adrenocortical tumors is the Weiss's system. ${ }^{7}$ According to this system, the presence of four or more of the nine criteria (high mitotic rate, atypical mitoses, high nuclear grade, and low percentage of clear cells, necrosis, diffuse tumor architecture, capsular invasion, sinusoidal invasion and venous invasion) indicates a malignant neoplasm (Table 2). ${ }^{8}$ 

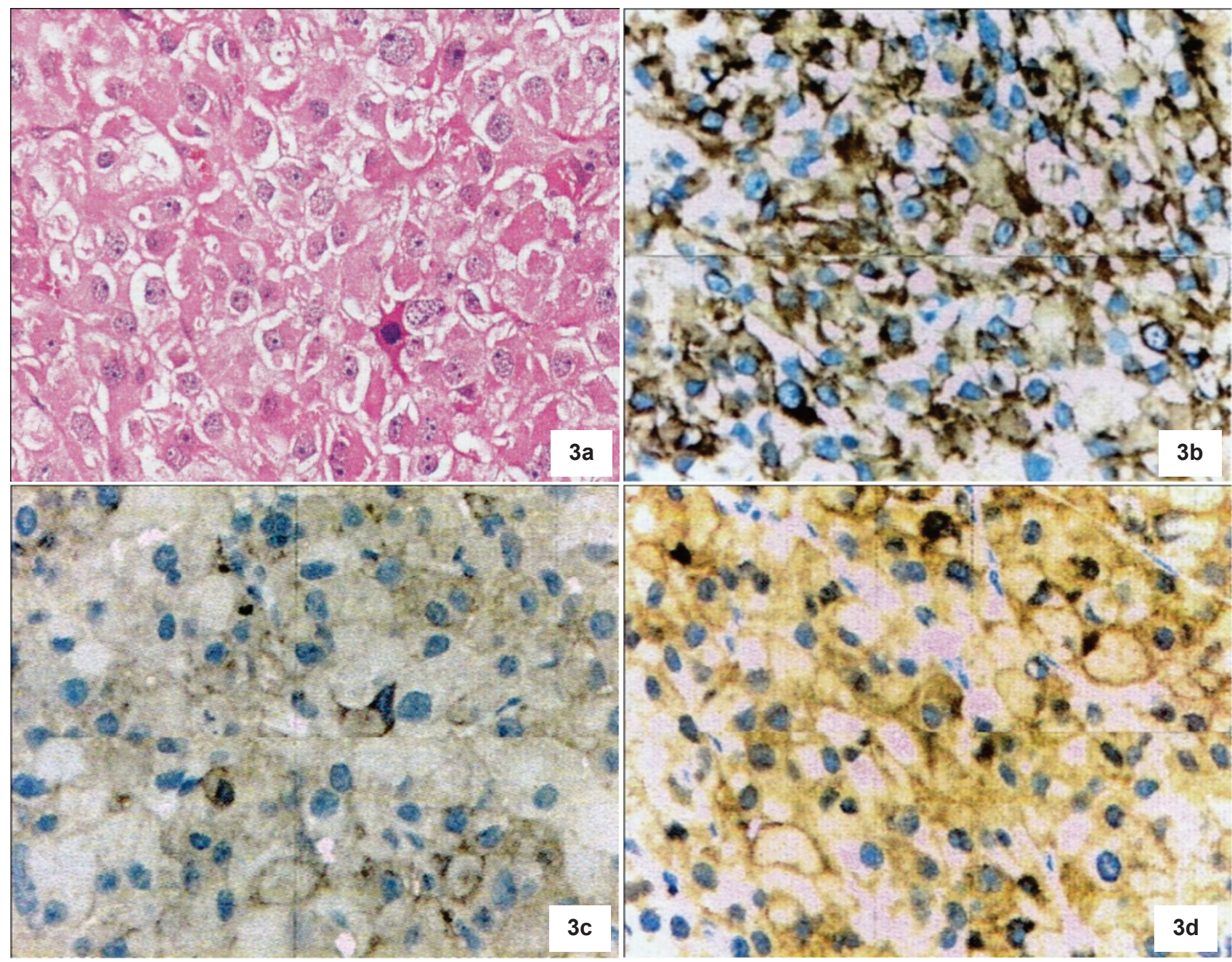

Figure 3a-d. 3a. The tumour is composed of polyclonal oncocytes with occasional mitosis (H\&E x 400). 3b. Strong positive cytoplasmic staining for vimentin in tumor cells.(IHC x 400). 3c. Weak positive cytoplasmic staining for synaptophysin (IHC $x$ 400). 3d. Strong positive cytoplasmic and cell membrane staining for neuron specific enolase (IHC x 400).

Table 2. Weiss histopathologic criteria of adrenal carcinoma ${ }^{9}$

\begin{tabular}{lc}
\hline \multicolumn{1}{c}{ Weiss system } & Score \\
\hline High nuclear grade & 1 \\
Mitotic rate more than 5/50 high power field & 1 \\
Atypical mitotic figures & 1 \\
Eosinophilictumor cell cytoplasm (>75\% of tumor cells) & 1 \\
Diffuse architecture (>33\% of tumor) & 1 \\
Necrosis & 1 \\
Venous invasion & 1 \\
Sinusoidal invasion (no smooth muscle in wall) & 1 \\
Capsular invasion & 1 \\
Total & 9
\end{tabular}

Total $0-2$ benign tumor

3 undetermined tumor

4-9 malignant tumor

*Weiss LM. Comparative histologic study of 43 metastasizing and nonmetastasizing adrenocortical tumors. Am J Surg Pathol. 1984; 8(3): 163-169.

Bisceglia et al, proposed new criteria with a modified form to classify the adrenocorticaloncocytic tumors. ${ }^{9}$ If the oncocytic tumor exhibits one or more of the major criteria (a mitotic rate of more than 5 mitoses per 50 high power fields, any atypical mitosis or any venous invasion), it is considered malignant. If the tumor exhibits one or more of the minor criteria such as large sized tumor $(>10 \mathrm{~cm}$ and/or >200 g), necrosis, capsular invasion, or sinusoidal invasion, the tumor is considered borderline (uncertain malignant potential). If none of the aforementioned features are present, the tumor can be considered benign. ${ }^{9}$ Although the weight of tumour, the presence of occasional atypical mitosis and few bizarre cells favor malignant potential, the absence of vascular and capsular invasion in our case suggests a benign process.

The biologic behavior of adrenocortical neoplasms is the most important practical problem. A combination of clinical, biochemical and histological features can differentiate benign and malignant adrenocortical tumors. Aside from the obviously malignant cases in which the diagnosis is based on common clinicopathologic features universally valid for tumors of any site, such as distant metastases, surgical unresectability and/or invasion of adjacent organs, the prediction of biologic behavior and clinical outcome can be difficult. ${ }^{10}$

Surgical resection remains the mainstay of the treatment. Although scoring systems exist in order to prognosticate tumour behavior, several uncertainties preclude accurate prediction of malignant behavior. Hence, a long term 
surveillance policy after surgical resection is advocated. ${ }^{2}$ In oncocytic carcinomas, radiotherapy, mitotane and/or chemotherapy is given post-excision, depending on disease staging and predominant symptoms. ${ }^{10}$

\section{CONCLUSION}

In conclusion, we present a rare case of feminizing adrenal oncocytoma in a man who was successfully operated. The malignant potential of this tumor is unpredictable and controversial. Nevertheless, we must pay attention and follow up frequently because little is known about the recurrence, behavior and biological characteristics of that tumor.

\section{References}

1. Juliano JJ, Cody RL, Suh JH. Metastatic adrenocortical oncocytoma: A case report. Urol Oncol. 2008; 26(2):198-201. http://dx.doi.org/ 10.1016/j.urolonc.2007.02.008.

2. Monk IP, Lahiri R, Sivaprakasam R, Malhotra S, Praseedom RK, Jaha A. Adrenocortical oncocytoma: Review of imaging and histopathological implications. Int J Surg Case Rep. 2010;1(3):30-32. http://dx.doi.org/10/1016/j.ijscr.2010.08.001.
1. El-Naggar AK, Evans DB, Mackay B. Oncocytic adrenal cortical carcinoma. Ultrastruct Pathol. 1991;15(4-5):549-56. http://dx.doi.org/ 10.3109/01913129109016262.

2. Sharma D, Sharma S, Jhobta A, Sood R. G. Virilizing Adrenal Oncocytoma. J Clin Imaging Sci. 2012;2(4):1-4. http://dx.doi.org/ 10.4103/2156-7514.104309.

3. Kabayegit OY, Soysal D, Oruk G, et al. Adrenocortical oncocytic neoplasm presenting with Cushing's syndrome: A case report. J Med Case Rep. 2008;2: 228-232. http://dx.doi.org.10.1186/1752-1947-2-228.

4. Kiriakopoulos A, Papaioannou D, Linos D. Adrenal cortical oncocytoma mimicking pheochromocytoma. Hormones.2011;10(1): 76-79.

5. Weiss LM, Medeiros LJ and Vickery LA Jr. Pathologic features of prognostic significance in adrenocortical carcinoma. American J Surg Pathol. 1989;13(3):202-206.

6. Medeiros LJ, Weiss LM. New developments in the pathologic diagnosis of adrenal cortical neoplasms. A review. Am J Clin Pathol. 1992;97:73-83.

7. Bisceglia M, Ludovico O, Mattia AD, et al. Adrenocortical oncocytic tumors: Report of 10 cases and review of the literature. Int J Surg Pathol. 2004;12(3):231-243. http://dx.doi.org/10.1177/106689690401200304.

8. Atmaca M, Yildiz S, Kalan I, et al. Functioning adrenocortical oncocytic carcinoma: A case report. Open Journal of Endocrine and Metabolic Diseases. 2013;3:42-45. http://dx.doi.org/10.4236/ ojemd.2013.31006.

Articles and any other material published in the JAFES represent the work of the author(s) and should not be construed to reflect the opinions of the Editors or the Publisher. Authors are required to accomplish, sign and submit scanned copies of the JAFES Declaration: that the article represents original material, that is not being considered for publication or has not been published or accepted for publication elsewhere. Consent forms, as appropriate, have been secured for the publication of information about patients; otherwise, authors declared that all means have been exhausted for securing such consent. The authors have signed disclosures that there are no financial or other relationships that might lead to a conflict of interest. All authors are required to submit Authorship Certifications that the manuscript has been read and approved by all authors, and that the requirements for authorship have been met by each author.

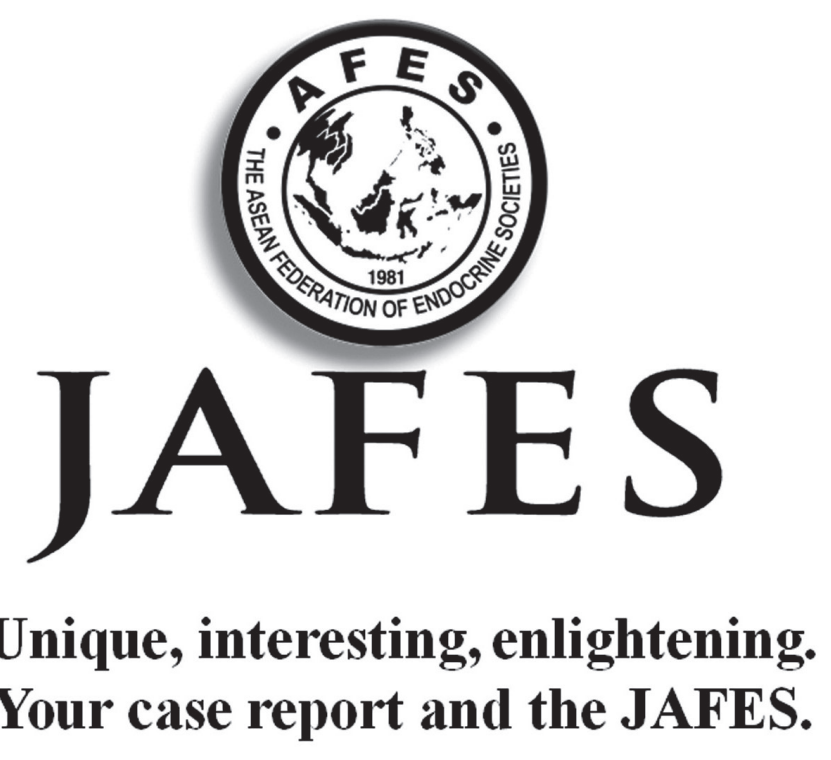

